\title{
Lebensmittel, Landwirtschaft, Lieferketten in der Krise: Verantwortung situieren
}

\section{Einleitung}

Die Covid-19-Pandemie macht die Fragilität von Lebensmittelversorgungssystemen sichtbar (vgl. IPES-Food 2020). Die aktuelle Krise löste eine neue Welle der Kritik an fehlender Resilienz von Produktions- und Distributionsstrukturen aus (vgl. ebd. Clapp 2020; Gustin 2020). Die Produktionskrisen im Globalen Norden und die sich beängstigend schnell abzeichnende Ernährungssicherheitskatastrophe in vielen Regionen des Globalen Südens sowie das Auftreten von Zoonosen überhaupt werden dabei zum Anlass genommen, eine Umkehr vom produktivistischen Paradigma (mehr, kostengünstiger, schneller) zu fordern (vgl. IPES-Food 2020; Clapp 2020; Vogel in Carstens 2020). Das Streben nach Effizienzsteigerung bringt Ökosysteme aus dem Gleichgewicht und verhindert dabei keine Versorgungslücken. Damit untergraben die gegenwärtigen Dynamiken die natürliche Grundlage der Agrarproduktion, ohne sich im akuten Krisenfall durch zuverlässig verfügbare Outputs auszuzeichnen. Resilienz - im Sinne der "anhaltenden Kapazität eines Lebensmittelversorgungssystems (...) im Angesicht verschiedener und sogar unvorhergesehener Störungen ausreichend angemessene und zugängliche Nahrung für alle zur Verfügung zu stellen « (Tendall et al. 2015: 19, eigene Übersetzung) sieht anders aus.

Forderungen nach Wandel bilden ein Transformationsmoment und gehen mit expliziten und impliziten Verantwortungszuschreibungen einher. Dabei sind die Verantwortungskonstellationen im Zusammenhang mit der aktuellen Krise in der Lebensmittelproduktion und -versorgung alles andere als eindeutig geklärt. Statt für eine eindeutige Klärung der Verhältnisse zu plädieren, nehmen wir diese Ambivalenzen zum Ausgangspunkt unserer Argumentation. Bei Lebensmittelversorgung handelt es sich nämlich um systemische Prozesse, deren Eigendynamik durch »Ungewissheit (...) und Unsicherheit « (Heidbrink 2017: 21) sowie komplexe Wechselwirkungen gekennzeichnet ist. Darüber hinaus konkurrieren in diesem Wirtschafts- und Handlungsfeld verschiedene Ideologien um die Deutungshoheit, wenn es um Kriterien für Resilienz und angemessene Zukunftsverantwortung geht (vgl. Curtis 2003; Pretty 2018).

In diesem Beitrag nehmen wir zunächst zwei designierte Verantwortungsträger*innen in den Blick: Konsument*innen und führende Unternehmen. Um auszuloten, wo Potenziale und Grenzen für einen Wandel bestehender Konfigurationen der Lebensmittelversorgung liegen, zeigen wir, auf welchen Wegen Verantwortung an sie herangetragen und von ihnen übernommen werden kann. Schließlich führen wir das Konzept der situierten Verantwortung ein und betonen die Signifi- 
kanz bestehender Verantwortungsbeziehungen. Das Konzept markiert eine mögliche Alternative, um über pauschale Verantwortungszuweisungen hinauszudenken. Gerade wenn es um Resilienz geht, so das Argument, ist ein kontextspezifischer Verantwortungsbegriff gefragt, der zwar keine Universallösungen verspricht, dafür aber an gegebene Belange und Stabilitäten anschließt. Damit verorten wir uns in einer sozial- und kulturanthropologischen Forschungsprogrammatik, welche die Untersuchung vielfältiger Verantwortungskonstellationen, deren Wechselwirkungen sowie Verhältnis zu zeitgenössischen Lebensweisen fokussiert (vgl. Trnka/Trundle 2014). Wir schließen den Beitrag mit einem Resümee zur Bedeutung von Verantwortungsbeziehungen im Kontext von Lebensmittelversorgungssystemen und einem Plädoyer für die Notwendigkeit, sie differenziert zu analysieren und zu mobilisieren. ${ }^{1}$

\section{Konsument*innen-Verantwortung}

Im Rahmen der eingangs erwähnten Forderungen nach einem Wandel der Lebensmittelproduktions- und -versorgungssysteme in Richtung Nachhaltigkeit und Resilienz ${ }^{2}$ gehören Endverbraucher*innen zu den am häufigsten adressierten Verantwortungssubjekten. Aus wirtschaftswissenschaftlicher Sicht bestimmen Konsument*innen maßgeblich über das Angebot (vgl. Schmidt 2017). Eine grundlegende Änderung der Wirtschafts- und Produktionsweisen bedürfe demnach in erster Linie eines signifikanten Richtungswechsels im Konsum. Ein solches Denkmodell steht letztlich auch im Einklang mit einer spezifischen Ethik, die häufig mit

1 Die zur Diskussion gestellten Überlegungen stützen sich auf sozialtheoretische Betrachtungen sowie entsprechend informierte langjährige Betrachtung von Diskursen um Ernährungspraktiken, Lebensmittelindustrie und Landwirtschaft. Diese fand im Kontext eines ethnographischen Forschungsprojektes von Ruzana Liburkina statt. Dabei wurden zwischen 03/2016 und 03/2018 58 Wochen Feldforschung, inklusive 56 Interviews, inmitten von acht unterschiedlichen Organisationskontexten in der Produktion und Distribution von Nahrungsmitteln durchgeführt. Entlang verschiedener Etappen der Wertschöpfungskette wurden eine transatlantische, konventionelle Reislieferkette und eine lokale, ökologische Getreide-zu-Brot-Lieferkette beforscht. Ein Teil unserer Argumentation speist sich aus dieser Empirie von Liburkina, auf die stellenweise Bezug genommen wird und die auf zentrale Dynamiken in der Lebensmittelversorgung verweist, welche durch die Pandemie aktueller und sichtbarer werden. Die Autorinnen arbeiten gemeinsam in der Forschungsinitiative >Configurations of Responsibilisation (CoRe) $<$ an der Goethe-Universität Frankfurt.

2 Während der Resilienzbegriff vor allem die Widerstandskraft und Adaptionsfähigkeit von Systemen im Angesicht von Krisenereignissen und unvorhergesehenen Bedrohungen beschreibt (vgl. Folke 2006), ist der Nachhaltigkeitsbegriff zukunfts- und kontinuitätsorientiert. Nachhaltige Systeme können langfristig fortbestehen, ohne dabei ihre eigenen Existenzvoraussetzungen zu gefährden. Im Zusammenhang mit sozioökologischen Systemen werden beide Eigenschaften als eng verflochten verhandelt (vgl. Maleksaedi/Karami 2013; Tendall et al.2015). 
dem Resilienzbegriff einhergeht. Diese Ethik stellt die Komplexität von Systemen als zentrales Verantwortungsproblem heraus. Reflexions- sowie Erkenntnisprozesse bezüglich der deshalb potenziell weitreichenden und unüberblickbaren Konsequenzen von Entscheidungen und Handlungen werden dabei als eine wichtige Kompetenz verantwortungsvoller Individuen verhandelt (vgl. Chandler 2013). Entsprechend nachdrücklich werden Konsument*innen mittels expliziter Apelle und Aufklärungskampagnen zum Umdenken und Reflektieren angeregt (vgl. BMU 2020; UBA 2020) oder implizit als Schlüsselfiguren verhandelt (vgl. WWF 2012).

Dass Endverbraucher*innen als Träger*innen von Verantwortung für die künftige Ausgestaltung der Lebensmittelversorgung angerufen werden, bedeutet zwar nicht, dass sie die ihnen zugeschriebene Verantwortung übernehmen können oder wollen. Die ethnographische Studie von Ruzana Liburkina zeigte jedoch, dass dieses Denkmodell enorme Wirkkraft in Produktions- und Handelskontexten entfaltet. ${ }^{3}$ Über diverse Stufen der Wertschöpfungskette hinweg ist ein großer Anteil von Personal- und Zeitressourcen der Erhebung und Evaluation von Nachfragetendenzen gewidmet. Auch dann, wenn es um übergeordnete Zielsetzungen wie Nachhaltigkeit geht, verorten sich vor allem kleine und mittelständische Unternehmen häufig in einer Abhängigkeitsposition, aus der heraus sie sich nach den Präferenzen von Konsument*innen richten müssten. Diese Einschätzung brachte ein Reisbauer aus Uruguay auf den Punkt, als er im Gespräch über Nachhaltigkeitszertifikate überspitzend anmerkte, dass diese eine wichtige Rolle spielen, da es die primäre Aufgabe von Produzent*innen sei, der »Hausfrau im europäischen Supermarkt" ein gutes Gewissen zu verschaffen (vgl. Feldnotiz Liburkina 12.10.2017).

Eine solche Aussage verdeutlicht, dass eine Argumentationslogik, die Endverbraucher*innen zu zentralen Verantwortungssubjekten stilisiert, die Handlungsgrundsätze und -bedingungen auf Produktionsseite prägt. Sie macht zugleich auf die Grenzen jener Logik aufmerksam, die gerade in Zeiten akuter Versorgungskrisen von großer Bedeutung sind. So mögen die Appelle zum bewussteren Lebensmittelkonsum durchaus einen Sinneswandel bei denjenigen Verbraucher*innen bewirken, die die bestehenden Problemlagen und ihre eigene systemische Rolle überblicken, an sich selbst als ethischen Subjekten arbeiten (vgl. Chandler 2013) und die notwendige Kaufkraft aufbringen können, um fair gehandelte und ökologisch produzierte Nahrungsmittel zu erwerben und damit immer mehr Produzent*innen zur Umstellung zu animieren. Ob das eine hinreichende Resilienzstrategie darstellt, ist allerdings fraglich. Die sozialwissenschaftliche Forschung zeigt, dass Konsument*innen keineswegs lückenlos informierte und reflektierte Akteur*innen sind, die gleichermaßen über das notwendige Wissen, die finanziellen Mittel und die Alltagsgewohnheiten verfügen, um einen ökologisch nachhalti-

3 Die entsprechende Dissertationsschrift wird im Jahr 2021 in Form einer englischsprachigen Monographie veröffentlicht. 
gen Ernährungsstil umzusetzen (vgl. Bourdieu 1982; John et al. 2016). Hinzu kommt, dass heute auch in der EU Teile der Bevölkerung unter anderem durch die Pandemie von Armut bedroht (vgl. EAPN Europe 2020) und daher weniger denn je in der Lage sind, einen höheren Preis für kurze Transportwege und ökologischen Landbau zu bezahlen. Viele Verbraucher*innen sind mehr denn je auf günstige Lebensmittel angewiesen. Bio-Produzent*innen, die es schaffen, Direktvertriebswege auszubauen, und einige neue reflektierte Konsument*innen erreichen (vgl. Kulms 2020), sind daher nur bedingt als Gewinner der Corona-Krise zu betrachten. Dass solche öko-lokalistischen Nischen (vgl. Curtis 2003) schrittweise global verteilte Produktions- und Handelsnetzwerke ablösen, scheint heute utopischer als vor der Pandemie. Besonders in der Krise sind es vor allem die ohnehin ressourcenstarken Akteure, die an Marktmacht gewinnen (vgl. Hobbs 2020; Rehse 2020). Daher sollten auch Verantwortungskonstellationen aktiviert werden, die über das Credo der Konsument*innen-Reflexivität und daran gekoppelte Nischen hinaus Resilienz und Nachhaltigkeit zu fördern vermögen.

\section{Unternehmensverantwortung}

Vor allem den >Big Playern und ihrer Marktmacht wird oft die zentrale Rolle für die Konsolidierung des Primats der Effizienz zugeschrieben (vgl. IPES-Food 2017; Lichtenstein 2006). So lässt die Preispolitik der großen Industrie- und Handelsunternehmen ihren Lieferanten kaum Spielräume in der Auswahl von Primärerzeugern. Ein Geschäftsführer eines mittelständischen Lebensmittelverarbeitungsunternehmens, das mehrere Einzelhandelsketten und Industriekonzerne beliefert, charakterisierte das Verhältnis zu diesen Firmen wie folgt: »Wir müssen die Hosen runterlassen, während der Andere noch seinen Schal umhat " (Interview Liburkina 18.07.2017). Seine Erzählung zeugt nicht von verlässlichen, durch Kooperation und Vertrauen gekennzeichneten Handelsbeziehungen, wie sie für Resilienz in Lieferketten als notwendig erachtet werden (vgl. Hobbs 2020). Dem Geschäftsmann zufolge sei es keine Ausnahme, sondern durchaus geläufig, dass viele Unternehmen fast oder ganz ohne Gewinnspanne arbeiten müssen, um dem Preisdruck in der Ausschreibung von Lieferanten standhalten zu können. So greifen lebensmittelverarbeitende Unternehmen häufig auf Produkte aus hochintensivierten Kulturlandschaften zurück, die unter einem hohen Einsatz an chemischen Dünge- und Pflanzenschutzmitteln ganzjährlich hohe Erträge mit geringen Qualitätsabweichungen liefern. Die Ausbreitung solcher Agrarlandschaften hat wiederum gravierende Folgen für Ökosysteme und kleinbäuerliche Strukturen (vgl. Foley et al. 2005; FAO 2019). Auch die durch die Corona-Krise wieder sichtbar gewordenen risikobehafteten Produktionsbedingungen in der konventionellen Fleischverarbeitungsindustrie resultieren letztlich aus dem von führenden Unternehmen gestützten Streben nach Effizienz (vgl. Striffler 2005). 
Wenn es um Nachhaltigkeit und Resilienz von Lebensmittelversorgungssystemen geht, gilt es also, den Preisdruck in seiner Funktion als gestaltende Kraft zu drosseln. Noch weniger als Konsument*innen lassen sich die Unternehmen, die in diesem Zusammenhang als vorrangig $\mathrm{zu}$ adressierende Verantwortungsträger identifiziert werden, allerdings durch Appelle erreichen. Vielmehr bewegen sich große Unternehmen - ob im Einzelhandel oder in der Industrie - in einem Regime der Zuschreibung und Übernahme von Verantwortung, welches auf dem Prinzip der Selbstkontrolle aufbaut (vgl. Power 1994) und durch das Phänomen der Corporate Social Responsibility (CSR) konstituiert wird. Der CSR-Begriff steht für ein breites Spektrum an Standards, Zertifizierungsschemata, Berichterstattungspflichten und Indikatoren, die das Verhältnis zwischen Unternehmen und ihren gesellschaftlichen und ökologischen Umwelten regulieren und transparent machen sollen (vgl. Garsten/Hernes 2009).

Heute werden sämtliche übergreifenden, gesellschaftlichen und ökologischen Belange, für die Firmen prospektiv Verantwortung übernehmen sollen, in dieses global und branchenübergreifend etablierte Regime integriert, indem sie in Indikatoren und Rechenschaftspflichten übersetzt werden. Wie die Einblicke aus der empirischen Studie von Liburkina zeigen, ist der Umgang mit CSR-Standards in der Lebensmittelbranche dabei weniger durch Reflexion, Abwägen von Normen und Einholen von Informationen, als vielmehr durch die Optimierung bürokratischer Routinen im Qualitätsmanagement gekennzeichnet. Nur wenn sich neu eingeführte Verantwortlichkeiten nicht reibungslos in die bereits etablierten Dokumentationsarchitekturen und Arbeitsabläufe integrieren ließen, wurden in den beforschten Handels-, Verarbeitungs- und Gastronomieunternehmen Entscheidungs- und Aushandlungsprozesse in Gang gesetzt (vgl. Liburkina 2019). Anderenfalls verlief die Übernahme von Verantwortung im Sinne von CSR automatisiert und ohne dass Urteilskraft, Kommunikations- und Handlungsfähigkeit als eigentliche Kriterien von Verantwortungsträgerschaft (vgl. Sombetzki 2014) überhaupt aufgerufen werden mussten.

Im Zusammenhang mit der Kopplung von Verantwortungsübernahme an die Vorzeigbarkeit und Standardisierung von Routinen und Organisationsstrukturen stellt die Corona-Krise ein besonderes Problem dar. Die neuen Herausforderungen in Arbeitssicherheit und Lebensmittelhygiene sowie die zwar diffusen, aber hohen gesellschaftlichen Erwartungen (vgl. IFH Köln 2020) binden eine Vielzahl von Ressourcen, um die Schwerpunkte in Qualitätssicherung und Öffentlichkeitsarbeit neu zu justieren. Durch die Flut an neuen, akut und unmittelbar krisenbedingten CSR-Handlungsanlässen (vgl. Shoup 2020) dürften weniger denn je Kapazitäten für die Bearbeitung langfristig verankerter, systemischer Probleme, wie etwa der aktuellen Ausrichtung von Beschaffungspraktiken, übrig bleiben. 


\section{Situierte Verantwortung für partikulare Andere}

Die im Zusammenhang mit der Gestaltung von Lebensmittelversorgungssystemen üblicherweise als Verantwortungssubjekte aufgerufenen Akteursgruppen - Konsument*innen und Unternehmen - sind im Kontext der Covid-19-Pandemie nur bedingt adressierbar. Mitunter sind sie unter den aktuellen Bedingungen noch weniger als sonst in der Lage, systemrelevanten Einfluss auf aktuell etablierte Wirtschaftsweisen auszuüben. Daraus folgt jedoch nicht, dass Covid-19 Verantwortungsträgerschaft vorbeugt oder ausschließt. Vielmehr macht die Krise sichtbar, dass die Diskurse rund um Konsument*innen- und Unternehmensverantwortung Systemeigenschaften, wie Resilienz, im Sinne von Verantwortungsgegenständen einordnen - »das Wofür? «(Sombetzki 2014: 32) von Verantwortung. Sowohl Konsument*innen als auch führende Unternehmen werden als verantwortlich für Resilienz aufgerufen, weil ihr Handeln systemische Auswirkungen hat. Die Covid-19-Pandemie macht außerdem sichtbar, dass solche Diskurse die praktischen Umstände und Bedingungen außer Acht lassen, unter denen die entsprechenden Akteur*innen Verantwortung übernehmen. Gerade in einer Krisensituation verändern sich diese Umstände und können die aus systemischer Sicht wünschenswerte Handlungsausrichtung dieser Schlüsselakteur*innen erschweren. Die Einordnung einzelner, generalisierter Akteursgruppen als Träger von Verantwortung für Resilienz im Sinne eines Verantwortungsgegenstandes mündet also in pauschalen Forderungen, denen es an situativer Kontextsensibilität mangelt.

In Anbetracht dieser Einsichten schlagen wir eine alternative Perspektive auf das Verhältnis zwischen Verantwortung und Resilienz im Zusammenhang mit Lebensmittelversorgungssystemen vor. Statt einer Förderung der Verantwortung für Resilienz plädieren wir für eine Förderung von Resilienz durch Verantwortung. Aus dieser Perspektive entsteht Resilienz dort, wo beständige, nicht von außen induzierte und nicht generalisierbare - situierte - Verantwortungsbeziehungen bestehen. Solche situierten Formen von Verantwortung in der Nahrungsmittelwirtschaft entstehen nicht rund um kontextübergreifende Ideale und Zielsetzungen. Dadurch unterscheiden sie sich unter anderem von der geteilten politischen Verantwortung, wie sie von Iris Marion Young (2011) konzeptualisiert wurde. Vielmehr handelt es sich dabei um eine kontextspezifische, notwendigerweise unvollständige und ignorante Form der Verantwortung, die sich auf ein ganz konkretes -Wir in expliziter Abgrenzung zu anderen Ideologien und Kollektivverständnissen bezieht. Diese situierte und nicht generalisierbare Form von Verantwortung wird in zahlreichen Verbindungen zwischen wirtschaftlichen Aktivitäten und Wissenspraktiken aktualisiert und nimmt darin Form an. In diesen Verbindungen werden privatwirtschaftliche Aktivitäten mit Bedeutung versehen, kontextualisiert, bewertet, gespiegelt und in ein ko-konstitutives Verhältnis zu anderen Praktiken und Akteur*innen sowie bestimmten Orten und Visionen gestellt.

Im Rahmen der ethnographischen Studie von Liburkina war situierte Verantwortung für partikulare Andere in zwei radikal unterschiedlichen Fällen beob- 
achtbar. So wurde die Rentabilität des Reissektors in Uruguay in dessen vielfältigen Verbindungen zu wirtschafts- und agrarwissenschaftlicher Expertise als essenziell für die Volkswirtschaft und deren Funktionsfähigkeit eingeordnet. Die engmaschige und hochgradig lokalisierte Beziehungs- und Ökosystempflege in einem alternativen Brot-Handelsnetzwerk in Deutschland wiederum wurde in Verbindungen zu alternativen Wissensformen aus der Ökonomie und Agrarökologie als politisch unentbehrlicher Widerstand gegen das konventionelle Lebensmittelversorgungssystem greifbar. Beide Einordnungen erfolgten, indem privatwirtschaftliches Agieren kontinuierlich in einen bestimmten Kontext gestellt und entsprechend gespiegelt und evaluiert wurde. Daraus erwuchsen auf Seiten der Landwirtschaft und Industrie langfristige Verpflichtungen und hartnäckige, überzeugte und als unanfechtbar verhandelte Positionierungen von Verantwortungssubjekten. Diese situierte Form von Verantwortungsträgerschaft entstand in beiden Fällen auf dem Fundament einer unauflösbaren Einbettung in bestimmte Repräsentationsordnungen und Diskurse und einer nicht verhandelbaren Zugehörigkeit zu einer bestimmten sozialen Welt. Sie zeichnete sich durch Beständigkeit aus und basierte auf Vertrauens- und Loyalitätsbeziehungen, kollektivem Gedächtnis und Wissenstransfer. Somit bezog sie sich zwar nicht auf Resilienz im Sinne eines Verantwortungsgegenstandes, entsprach aber selbst den Kriterien sozialer Resilienz (vgl. Adger 2003; Djalante et al. 2013).

Beide hier exemplarisch vorgestellten Formen situierter Verantwortung sind jeweils unvollständig und bewirken keinen Strukturwandel. Daher stellen diese Formen der situierten Verantwortung für sich allein genommen noch keine >Blaupausen ‘ für Resilienz und Nachhaltigkeit von Lebensmittelversorgungssystemen dar. Nichtsdestotrotz kann darin ein wichtiger Schlüssel für entsprechende Bemühungen liegen. Bereits vorhandene und robuste Positionierungen von Verantwortungssubjekten lösen nämlich Kommunikationsprobleme, die im Zuge solcher Bestrebungen eine Rolle spielen (vgl. Meckenstock et al. 2016). Träger*innen situierter Verantwortung für partikulare Andere, die sich ohnehin als solche begreifen, lassen sich durchaus gezielt adressieren und gehen Reflexionsprozesse ein. Für sie steht im Zusammenhang mit Lebensmittelproduktion etwas auf dem Spiel, das sich nicht auf Profitmaximierung oder abstrahierte Normvorstellungen reduzieren lässt.

Unser Fokus auf situierte Verantwortung im Zusammenhang mit Lebensmittelproduktion und -distribution bestätigt die Annahme, dass der Schlüssel für die Förderung krisenresistenter und lernfähiger Lebensmittelproduktions- und -versorgungssysteme im geteilten Sinn für Verantwortung liegt (vgl. Tiensin et al. 2020). Der vorliegende Beitrag konkretisiert diese Annahme dabei insofern, als wir anregen, bereits vorhandenen, kontextspezifischen und nicht aufzulösenden Verantwortungsbeziehungen mehr Aufmerksamkeit zu widmen. Dabei müssen nicht nur alternative Produktionsnischen mobilisiert werden. Auch in konventionellen, industrialisierten Kontexten verdienen konstante Anbindungen und Ver- 
antwortungsbeziehungen vonseiten privatwirtschaftlicher Akteur*innen besondere Beachtung.

Die fehlende Resilienz von Lebensmittelversorgungssystemen ist kein einfaches, linear zu lösendes, sondern ein komplexes, >vertracktes‘ Problem ${ }^{4}$. Die Lösungsbemühungen münden somit nicht in Konsens, sondern unweigerlich in divergenten Diskursen (vgl. King 1993). Werden die bestehenden Verpflichtungen und Anbindungen von Akteur*innen in ihrer Divergenz und Unvollständigkeit wahr- und ernstgenommen, sind sie als Verantwortungssubjekte adressierbar und in der Lage, Reflexionsprozesse einzugehen und an Transformationsagenden mitzuwirken. Die akute Produktions- und Versorgungskrise, die durch die globale Ausbreitung des Covid-19-Virus ausgelöst wurde, stellt ein wichtiges Transformationsmoment dar, weil sie sämtliche Akteur*innen in der Nahrungsmittelproduktion tangiert. Eine dezidierte Berücksichtigung und Mobilisierung situierter Verantwortung ist wichtig, um dieses Transformationsmoment trotz kontrastierender Agenden als solches fruchtbar machen zu können. Bislang konzentriert sich Regierungshandeln im Zuge der Auslotung möglicher Resilienzstrategien in akuten Krisen vor allem darauf, Multi-Stakeholder-Kollektive ${ }^{5}$ einzurichten (vgl. SinghPeterson et al. 2015; UN Global Compact 2020). Obgleich solche Initiativen geteilte Verantwortung institutionalisieren sollen, werden sie mitunter im Sinne eines neoliberalen Delegierens von Verantwortung kritisiert (vgl. Welsh 2014) und verhindern nicht, dass Verantwortung in erster Linie regierenden Akteur*innen zugeschrieben wird (vgl. Singh-Peterson et al. 2015). Um diese Schwachstellen induzierter kollektiver Verantwortung zu kompensieren, sollten verstärkt komplementäre Reflexions- und Dialogprozesse initiiert werden, die auf bestehenden Verantwortungskonstellationen aufbauen. So könnten bereits durch situierte geteilte Verantwortung verbundene Akteur"innen aus der Lebensmittelwirtschaft zusammenfinden, um partikulare, aber robuste Antworten auf die Frage zu formulieren, die heute alle beschäftigt: Wie kann und soll die Zukunft nach Covid-19 gestaltet werden?

\section{Fazit}

Die Covid-19-Pandemie ist nicht nur eine globale Krise der öffentlichen Gesundheit, sondern auch des Lebensmittelsektors. Daraus resultiert die Frage, wie dieser resilienter und nachhaltiger werden kann. Im Zuge von Forderungen nach einem

4 Für eine umfassende Definition von >wicked problems teristika, die sie auszeichnen, vgl. Rittel/Webber 1973.

5 Multistakeholder-Netzwerke sind »Initiativen (...), bei denen Nichtregierungsorganisationen (NRO), Gewerkschaften, Unternehmen und (...) [Regierungsvertreter*innen; R. L./L. O.] für eine Branche oder ein Produkt gemeinsame Lösungsansätze zur Stärkung der menschenrechtlichen und ökologischen Verantwortung entlang der Lieferkette suchen «(CorA et al, 2017:2). 
solchen Wandel werden zum einen die Endverbraucher*innen zu Verantwortungssubjekten erklärt, zum anderen wird die Verantwortung großer Unternehmen diskutiert. Aus kulturanthropologischer und sozialwissenschaftlicher Perspektive erörterten wir, warum Aufklärung und Appell unzureichend sind, um einen langfristigen Wandel in der globalen Lebensmittelversorgung zu bewirken. Im Hinblick auf Großunternehmen haben wir diskutiert, dass sie zwar eine Schlüsselrolle bei der Gestaltung von Lieferketten innehaben, allerdings in erster Linie mittels formalisierter Verantwortungszuschreibungen adressiert werden können. Den pauschalisierten Zuschreibungen von Verantwortungsträgerschaft an bestimmte Akteur*innengruppen haben wir schließlich das Konzept der situierten Verantwortung für Andere entgegengestellt. Dieser kontextspezifische Verantwortungsbegriff bildet die Grundlage für Verantwortungszuschreibungen, die universelle Ansprüche zugunsten der Stärkung vorhandener Beziehungs- und Verpflichtungsnetzwerke aufgeben.

Wie also weiter vor diesem Hintergrund und im Kontext von >Lehren aus Corona<? In Zeiten einer komplexen Versorgungskrise gilt es zunächst, das Streben nach Effizienz durch ein Streben nach Resilienz abzulösen (vgl. De Schutter in Dundas et al. 2020). Dabei sind Lösungen gefragt, die an diejenigen Strukturen, Belange, Notwendigkeiten und Selbstverständlichkeiten anschließen, die dieses Wirtschaftsfeld und dessen Unzulänglichkeiten aktuell prägen. Welche Akteur*innen auf welche Weisen Verantwortung übernehmen können, ist demzufolge eine Frage, die einer detaillierten Auseinandersetzung mit bestehenden Handlungsbedingungen und strukturellen Voraussetzungen bedarf. Während Interventionen vor allem mittels Rechts- und politischer Steuerungsinstrumente erfolgen müssen, kann empirische, nahe an den relevanten Akteur*innen ausgerichtete Sozialforschung wertvolle Einblicke in ebenjene Ausgangsbedingungen liefern.

Wenn das Ziel ist, Ernährungssicherheit langfristig, zuverlässig und global gerecht zu gewährleisten, sollte ein Verständnis davon generiert werden, welche Akteur*innen wie adressierbar sind und wie nicht. Nur so können sinnvolle, an konkrete Gegebenheiten angepasste »strukturelle Vermittlungen konzipiert werden, >reflexive Arrangements‘, die den Selbstlauf der Systeme unterbrechen und dadurch Verantwortung möglich machen« (Wilhelms 2017: 522). Genau diese Art der gerichteten Unterbrechung verhärteter Produktions- und Distributionsmuster ist wiederum notwendig, um die Lehren aus der Covid-19-Pandemie für eine nachhaltigere Zukunft der Lebensmittelversorgung zu mobilisieren. ${ }^{6}$

6 Das ethnographische Forschungsprojekt, auf das dieser Beitrag Bezug nimmt, wurde von der Deutschen Bundesstiftung Umwelt gefördert. Darüber hinaus gilt unser Dank einer*m anonymen Gutachter*in für wertvolle Hinweise zu einer früheren Version dieses Aufsatzes. 


\section{Literaturverzeichnis}

Adger, W. N. (2003): Social Capital, Collective Action, and Adaptation to Climate Change, in: Economic Geography, Vol. 79/No. 4, 387-404.

BMU (2020): Nachhaltiger Konsum. Bundesministerium für Umwelt, Naturschutz und nukleare Sicherheit, Stand 14.04.2020. Link: https://www.bmu.de/themen/wirtschaft-prod ukte-ressourcen-tourismus/produkte-und-konsum/nachhaltiger-konsum/ (last access on May 28th, 2020).

Bourdieu, P. (1982): Die feinen Unterschiede. Kritik der gesellschaftlichen Urteilskraft, Frankfurt/Main: Suhrkamp.

Carstens, P. (2020): Naturforscher über COVID-19: »Alles, was wir Menschen tun, kommt zu uns zurück ", in: GEO, 26.03.2020. Link: https://www.geo.de/natur/nachhaltigkeit/22797rtkl-corona-pandemie-naturforscher-ueber-covid-19-alles-was-wir-menschen (zuletzt abgerufen am 28.05.2020).

Chandler, D. (2013): Resilience Ethics: Responsibility and the Globally Embedded Subject, in: Ethics \& Global Politics, Vol. 6/No. 3, 175-194.

Clapp, J. (2020): Spoiled Milk, Rotten Vegetables and a Very Broken Food System, in: The New York Times, 08.05.2020. Link: https://www.nytimes.com/2020/05/08/opinion/coron avirus-global-food-supply.html (last access on May 28th, 2020).

CorA/Forum Menschenrechte/Forum Umwelt und Entwicklung/VENRO/Verbraucherzentrale Bundesverband (2017): Multistakeholder-Initiativen: Grenzen und Voraussetzungen aus Sicht der Zivilgesellschaft. CorA-Netzwerk für Unternehmensverantwortung. Link: https:// www.cora-netz.de/wp-content/uploads/2017/09/2017-09_MSI_Positionspapier_CorA-FM R-FUE-VENRO-vzbv_web.pdf (zuletzt abgerufen am 28.05.2020).

Curtis, F. (2003): Eco-localism and Sustainability, in: Ecological Economics, Vol. 46/No. 1, 83-102.

Djalante, R./Holley, C./Thomalla, F./Carnegie, M. (2013): Pathways for Adaptive and Integrated Disaster Resilience, in: Natural Hazards, Vol. 69/No. 3, 2105-2135.

Dundas, M./Bertsch, M./Guggenheim, J. (2020): Covid-19: Global Food Supply on a Razor's Edge, in: France 24, Down to Earth, 11.05.2020. Link: https://www.france24.com/en/202 00511-down-to-earth-covid-19-global-food-supply-on-a-razor-s-edge (last access on May 28th, 2020).

EAPN Europe (2020): Millions of People's Jobs, Income and Living Standards are at Risk, in: The European Anti-Poverty Network, 17.03.2020. Link: https://www.eapn.eu/eapn-statem ent-on-covid-19/ (last access on May 28th, 2020).

FAO (2019): The State of the World's Biodiversity for Food and Agriculture. Rome: FAO Commission on Genetic Resources for Food and Agriculture Assessments. Link: http://ww w.fao.org/3/CA3129EN/CA3129EN.pdf (last access on May 28th, 2020).

Foley, J. A./Defries, R./Asner, G. P./Barford, C./Bonan, G./Carpenter, S. R./Chapin, F. S./Coe, M. T./Daily, G. C./Gibbs, H. K./Helkowski, J. H./Holloway, T./Howard, E. A./Kucharik, C. J./Monfreda, C./Patz, J. A./Prentice, I. C./Ramankutty, N./Snyder, P. K. (2005): Global Consequences of Land Use, in: Science, Vol. 309/No. 5734, 570-574.

Folke, C. (2006): Resilience: The Emergence of a Perspective for Social-Ecological Systems Analyses, in: Global Environmental Change, Vol. 16/No. 3, 253-267.

Garsten, C./Hernes, T. (2009): Beyond CSR: Dilemmas and Paradoxes of Ethical Conduct in Transnational Organizations, in: Browne, K. E./Milgram, B. L. (Eds.): Economics and Morality: Anthropological Approaches, Lanham, New York, Toronto, Plymouth: AltaMira Press, 189-210.

Gustin, G. (2020): Empty Grocery Shelves and Rotting, Wasted Vegetables: Two Sides of a Supply Chain Problem, in: Inside Climate News, 19.04.2020. Link: https://insideclimatene 
ws.org/news/17042020/coronavirus-agriculture-supply-chain-grocery-store-farming (last access on May 28th, 2020).

Heidbrink, L. (2017): Definitionen und Voraussetzungen der Verantwortung, in: Heidbrink, L./ Langbehn, C./Loh J. (Hrsg.): Handbuch Verantwortung, Wiesbaden: Springer VS, 333.

Hobbs, J. E. (2020): Food Supply Chains during the COVID-19 Pandemic, in: Canadian Journal of Agricultural Economics/Revue canadienne d'agroeconomie, Vol. 68/No. 2, 171177.

IFH Köln (2020): Corona Consumer Check: Handelsunternehmen kommt große Verantwortung zu, in: IFH Köln Information, Research und Consulting, 02.04.2020. Link: https://w ww.ifhkoeln.de/pressemitteilungen/details/corona-consumer-check-handelsunternehmen-k ommt-grosse-verantwortung-zu/ (zuletzt abgerufen am 28.05.2020).

IPES-Food (2017): Too Big to Feed: Exploring the Impacts of Mega-Mergers, Concentration, Concentration of Power in the Agri-Food Sector. Link: http://www.ipes-food.org/_img/upl oad/files/Concentration_FullReport.pdf (last access on May 28th, 2020).

IPES-Food (2020): Covid-19 and the Crisis in Food Systems: Symptoms, Causes, and Potential Solutions. Communiqué by International Panel of Experts on Sustainable Food Systems. Link: http://www.ipes-food.org/_img/upload/files/COVID-19_CommuniqueEN\%28 $3 \% 29$.pdf (last access on May 28th, 2020).

John, R./Jaeger-Erben, M./Rückert-John, J. (2016): Elusive Practices: Considerations on Limits and Possibilities of Environmental Policy for Sustainable Consumption, in: Environmental Policy and Governance, Vol. 26/No. 2, 129-140.

King, J. B. (1993): Learning to Solve the Right Problems: The Case of Nuclear Power in America, in: Journal of Business Ethics, Vol. 12/No. 2, 105-116.

Kulms, J. (2020): Biolebensmittel-Lieferservice: Boom in der Coronakrise, in: Deutschlandfunk, 07.04.2020. Link: https://www.deutschlandfunk.de/biolebensmittel-lieferservice-boom-inder-coronakrise.1769.de.html?dram:article_id=474253 (zuletzt abgerufen am 28. Mai 2020).

Liburkina, R. (2019): Verantwortungsübernahme praxistheoretisch gedacht: Verhältnis von Handlungs- und Verantwortungsträgerschaft in der Auditkultur, in: RphZ - Rechtsphilosophie - Zeitschrift für Grundlagen des Rechts, Vol. 5/No. 2, 170-184.

Lichtenstein, N. (Hrsg.) (2006): Wal-Mart: The Face of Twenty-First-Century Capitalism, New York, London: The New Press.

Maleksaeidi, H./Karami, E. (2013): Social-Ecological Resilience and Sustainable Agriculture Under Water Scarcity, in: Agroecology and Sustainable Food Systems, Vol. 37/No. 3, 262290.

Meckenstock, J./Barbosa-Póvoa, A. P./Carvalho, A. (2016): The Wicked Character of Sustainable Supply Chain Management: Evidence from Sustainability Reports, in: Business Strategy and the Environment, Vol. 25/No. 7, 449-477.

Power, M. (1994): The Audit Explosion, London: Demos.

Pretty, J. (2018): Intensification for Redesigned and Sustainable Agricultural Systems, in: Science, Vol. 362/No. 6417, 1-7.

Rehse, A. (2020): Supermarktketten diktieren Preise, in: SWR, 26.03.2020. Link: https://www. swr.de/wissen/odysso/supermarktketten-diktieren-preise-100.html (zuletzt abgerufen am 28.05.2020).

Rittel, H. W. J./Webber, M. M. (1973): Dilemmas in a General Theory of Planning, in: Policy Sciences, Vol. 4/No. 2, 155-169.

Schmidt, I. (2017): Konsumentenverantwortung, in: Heidbrink, L./Langbehn, C./Loh J. (Hrsg.): Handbuch Verantwortung, Wiesbaden: Springer VS, 735-764. 
Shoup, M. E. (2020): Big Food and Coronavirus: The Response so far, in: FoodNavigatorUSA, 06.04.2020. Link: https://www.foodnavigator-usa.com/Article/2020/04/06/Big-Food -and-coronavirus-The-response-so-far\# (last access on May 28th, 2020).

Singh-Peterson, L./Salmon, P./Baldwin, C./Goode, N. (2015): Deconstructing the Concept of Shared Responsibility for Disaster Resilience: A Sunshine Coast Case Study, Australia, in: Natural Hazards, Vol. 79/No. 2, 755-774.

Sombetzki, J. (2014): Verantwortung als Begriff, Fähigkeit, Aufgabe: Eine Drei-Ebenen-Analyse, Wiesbaden: Springer Fachmedien.

Striffler, S. (2005): Chicken: The Dangerous Transformation of America's Favorite Food, New Haven, London: Yale University Press.

Tendall, D. M./Joerin, J./Kopainsky, B./Edwards, P./Shreck, A./Le, Q. B./Kruetli, P./Grant, M./Six, J. (2015): Food System Resilience: Defining the Concept, in: Global Food Security, Vol. 6, 17-23.

Tiensin, H./Kalibata, A./Cole, M. (2020): Ensuring Food Security in the Era of COVID-19, in: United Nations Sustainable Development Goals, 08.04.2020. Link: https://www.un.org/su stainabledevelopment/blog/2020/04/ensuring-food-security-covid-19/ (last access on May 28th, 2020).

Trnka, S./Trundle, C. (Eds.) (2017): Competing Responsibilities: The Politics and Ethics of Contemporary Life, Durham, London: Duke University Press.

UBA (2020): Kompetenzzentrum Nachhaltiger Konsum, in: Umweltbundesamt, 22.08.2019. Link: https://www.umweltbundesamt.de/themen/wirtschaft-konsum/kompetenzzentrum-na chhaltiger-konsum (zuletzt abgerufen am 28.05.2020).

UN Global Compact (2020): UN Global Compact Defines New Level of Ambition for Corporate Sustainability, in: United Nations, 09.06.2020. Link: https://www.unglobalcompact.o rg/news/4575-06-09-2020 (last access on June 18th, 2020).

Welsh, M. (2014): Resilience and Responsibility: Governing Uncertainty in a Complex World, in: The Geographical Journal, Vol. 180/No. 1, 15-26.

Wilhelms, G. (2017): Systemverantwortung, in: Heidbrink, L./Langbehn, C./Loh J. (Hrsg.): Handbuch Verantwortung, Wiesbaden: Springer VS, 501-524.

WWF (2012): Klimawandel auf dem Teller. Link: https://mobil.wwf.de/fileadmin/fm-wwf/Publi kationen-PDF/Klimawandel_auf_dem_Teller.pdf (zuletzt abgerufen am 28.05.2020).

Young, I. M. (2011): Responsibility for Justice, New York: Oxford University Press. 